\title{
INTEGRATEd OPTICAL DUAL MACH-ZEHNDER INTERFEROMETER SENSOR
}

\author{
Ping Hua, B. Jonathan Luff*, Geoffrey R. Quigley \& James S. Wilkinson** \\ Optoelectronics Research Centre, University of Southampton, Southampton, Hampshire \\ SO17 1BJ, UK \\ \&
}

Kenji Kawaguchi

Kyoto Electronics Manufacturing Co., Ltd., 68 Ninodan-cho, Shinden, Kisshoin, Minami-ku, Kyoto 601-8317, Japan

\begin{abstract}
The fabrication and operation of robust integrated optical refractometers, suitable for precise measurements of small changes without ambiguity over a wide range of refractive indices, is described. The design primarily uses an optical fibre coupled dual-sensitivity integrated optical Mach-Zehnder Interferometer sensor chip incorporating 3x3 directional coupler combiners and internal referencing. High-index $\mathrm{Ta}_{2} \mathrm{O}_{5}$ films were deposited on the waveguide surface in order to increase sensitivity and measurements of their response to liquid analyte index have been carried out by passing aqueous sucrose solutions over the sensor surface. These devices are intended for application as high-sensitivity multi-purpose chemical sensors and biosensors.
\end{abstract}

Keywords: Integrated optics, Sensors, Instrumentation, Interferometers

* Now with Bookham Technology plc, 90 Milton Park, Abingdon, Oxfordshire, OX14 4RY, UK

** Corresponding author: Tel: +44 (0)23 8059 2792; Fax: +44 (0)23 8059 3149;

Email:jsw@orc.soton.ac.uk 


\section{Introduction}

Integrated optical transducers for real-time measurement of interactions between biological molecules, and for the specific detection of chemical and biochemical species, are the subject of growing interest [1]. Integrated optics allows high detection sensitivity to be achieved using optical transduction techniques in a compact and robust format. Applications of this technology include environmental pollution monitoring, industrial process control and medical diagnostics. The increasing complexity of such applications demands integration, so that approaches that allow the interrogation of arrays of sensors on microstructured surfaces, where small volumes of analyte are controlled by integrated microfluidic systems, are expected to find wide application. Planar optical waveguides embedded in flat dielectric substrates may be employed to guide light at a surface on which films sensitised to specific chemicals have been immobilised, allowing real-time interrogation of their optical properties during chemical reactions. This approach offers precise stable control of the optical interaction, reduction in interference from the bulk of the analyte, and potential for photolithographic production of dense sensor arrays.

The wide range of optical phenomena which may be used to observe reactions in thin films at surfaces has prompted the development of sensor devices based upon the simple low-cost base technology of ion-exchange in glass [2-5]. The intrinsic sensitivity of these waveguides to surface effects is lower than for waveguides formed from high-index thin films [6,7] due to their reduced optical confinement or larger modal dimensions. However they exhibit low loss, are well-suited to photolithographic formation of monomode channel waveguides, may readily be connected to instrumentation in a stable manner using pigtailed optical fibres, and the surface evanescent fields may be locally enhanced at sensing sites by incorporation of conducting [8] or dielectric [9] overlayers. The permanent connection of optical fibres is relatively costly when compared with "free-space" coupling of light into thin-film 
waveguides with gratings but brings the advantages of mechanical stability and ease of control of the spatial distribution of the evanescent intensity over the surface of the "chip". This approach is not aimed at very cheap "single-shot" devices but at arrays of sensors for multiple analytes, where the surface chemistry may be regenerated in situ using electrochemical or chemical regeneration allowing repeated use.

The integrated optical Mach-Zehnder interferometer (MZI) is an evanescent refractometer whose surface may be chemically modified to render it sensitive to specific chemical species. In principle the MZI is more sensitive than waveguide surface plasmon resonance (SPR) based sensors [6], primarily because it does not employ coupling to a lossy waveguide, although exploitation of this enhanced sensitivity may require the sensitive region to be longer. Furthermore, the MZI may be rendered sensitive over a wider range of superstrate indices, as it does not employ "resonant" coupling between two dissimilar waveguides but, in effect, directly measures modal velocity changes due to changes in refractive index in the evanescent field of an unisolated waveguide compared with a waveguide isolated from the analyte. Highly sensitive MZI immunosensors have already been demonstrated, for example by Heideman et al. [7], and our work has concentrated upon achieving sensitive operation over a wide range of indices, enhancing the sensitivity of ionexchanged devices using high-index overlayers [9], and investigating the simple and reliable incorporation of these sensors into instrumentation.

The aim of the present approach is to realise an integrated refractometer chip for incorporation in practical instrumentation, for measurements in analytes having a wide range of indices. The Mach-Zehnder interferometer has been chosen as it is straightforward to design and fabricate, and is tolerant of manufacturing error. The design adopted incorporates i) thin tantalum pentoxide films to enhance sensitivity, ii) a high-sensitivity MZI and a low- 
sensitivity MZI to allow the combination of high sensitivity with wide index range, iii) $3 \times 3$ output couplers from each MZI to ensure sensitive operation over the entire index range and to remove ambiguity in the direction of index change, and iv) reference waveguides with and without analyte windows, to allow compensation for the effects of input power fluctuations and potentially to determine analyte absorption. Incorporation in practical instrumentation demands that inexpensive and reliable techniques for addressing the multiple outputs of waveguide devices must be found. Fibre-to-chip pigtailing of integrated optical devices formed in 'passive' materials such as glass, where it is difficult to truly integrate monolithic light sources and detectors, is not the best solution when dealing with multiple outputs due to the necessity of producing and pigtailing fibre arrays. For single input devices, however, fibre input coupling is still a viable option as only a single pigtail needs to be made.

In this paper we present multiple-output integrated optical sensor devices using fibre input coupling and a cheap, readily available, 1D CCD array detector to simultaneously address all outputs. This approach leads to a low-cost reconfigurable approach to the simultaneous acquisition of multiple outputs. A lens is used to focus the waveguide outputs onto the array, resulting in a compact unit that can be housed in a standard instrument package. A further advantage of this arrangement is that other optical elements such as filters and polarisers can readily be inserted into the beam path. Results are presented for measurements on bulk analytes and on real-time measurement of binding of biological molecules to an immobilised film. Work is presently in progress to precisely stabilise the temperature of the sensor surface through feedback control and to explore the use of selective polymers in sensor arrays for quantification of chemical species of interest in beverage analysis. 


\section{Transducer operation}

A schematic diagram of a typical device and outline measurement system is shown in Figure 1. Light is coupled into the sensor chip using a monomode fibre and the power is divided equally into a "left branch" which feeds the two interferometers and a "right branch" for referencing purposes. Power in the "left branch" is split again to feed the two interferometers and then again into the two arms of each interferometer. In each interferometer, the relative phase of the light in the two paths combining at the $3 \times 3$ coupler depends upon the refractive index of the analyte in the window in the isolation layer which otherwise covers the entire device. One MZI has a window ten times shorter than the other, resulting in a sensitivity ten times lower, to remove ambiguities due to the periodic nature of the response to index for large index changes. In a conventional MZI the light is recombined in a simple Y-junction, resulting in an output which varies periodically with relative phase, with excess power being scattered into the substrate. Sensitive transducer response to small changes in analyte index requires that the device must always operate in a sensitive region of the response curve. This cannot be satisfied over the entire range of indices for a singleoutput MZI, which shows maxima and minima in the interference function. The use of a three-waveguide coupler results in three outputs, with interference functions shifted by nominally $120^{\circ}$ with respect to each other. In this way at least one output yields a sensitive response to small index changes whatever the baseline index [10]. While use of $3 \times 3$ couplers tightens the constraints on manufacture somewhat, it also allows referencing the individual signals to the sum of the signals emerging from the three outputs, assuming negligible variation in total loss as power is distributed among them, removing the effects of source intensity fluctuations.

Two reference outputs are provided; the first is isolated from the analyte for its entire length, allowing simple removal of the effects of input power fluctuations and to eliminate the 
effects of analyte absorption. In the presence of optically absorbing analyte media this reference output may be used to estimate the optical absorption of the analyte as well as its refractive index. The second reference waveguide is exposed to the analyte through an identical window to that of the high-sensitivity MZI, yielding a direct measurement of the loss due to the window and analyte. The whole chip may be coated with a thin film of a robust high-index material such as tantalum pentoxide in order to increase the sensitivity of both MZIs and the exposed reference waveguide.

\section{Design and fabrication of MZI chips}

3.1 Ion-exchanged waveguides. Two transducer chips, as shown in Figure 1, were fabricated by potassium ion-exchange in BK7 glass [11]. This substrate glass has more reproducible characteristics than the soda-lime glass used in earlier work [9] and has a refractive index of approximately 1.51 at the wavelength of interest, allowing measurements on analytes of index up to approximately 1.5. The index range required for our applications is between 1.32 and 1.41, which is readily achievable using this waveguide system. An aluminum masking film was deposited on each substrate and the waveguide circuit was defined by tracks $1.5 \mu \mathrm{m}$ wide opened in it using conventional photolithography. The threeoutput couplers, designed for a wavelength of $635 \mathrm{~nm}$, had a coupling length of $0.5 \mathrm{~mm}$, and the waveguide centres were separated by $3.6 \mu \mathrm{m}$. Ion exchange was carried out by immersing the masked substrates in $\mathrm{KNO}_{3}$ at $400^{\circ} \mathrm{C}$ for $2 \mathrm{hrs}$ and the ends of the chips were then polished to allow input and output coupling, resulting in an overall length of approximately $44 \mathrm{~mm}$.

3.2 Isolation layer. Vacuum-deposited Teflon FEP has proved a useful isolation material for experimental devices [10], due to its low refractive index, low porosity and ease of patterning, but it has not so far proved acceptable for use in sensors which are to be handled 
repeatedly, as it is easily damaged. Development of these devices for prolonged use in an instrument requires optimisation of hard dielectric materials, such as sputtered silica films, as robust isolation layers. However, sputtered silica tends to exhibit higher optical attenuation than vacuum-evaporated Teflon, unless it is annealed in an oxygen-bearing atmosphere at high temperature. As the silica in these devices is to be deposited on substrates containing ion-exchanged surface waveguides, it is important to determine an acceptable deposition process without high-temperature annealing which would degrade the underlying waveguides by thermal diffusion. Prior to fabrication of the sensor chips, the silica film deposition process was optimised by sputtering silica films on similar potassium ion-exchanged waveguides under a variety of conditions, and then annealing at $250^{\circ} \mathrm{C}$ in oxygen. Figure 2 shows the transmission of 15 waveguides relative to an uncoated waveguide immediately after deposition and after annealing for 1, 2 and 4 hours, numbers 17 being coated with a $10 \mathrm{~mm}$ length of silica and numbers $8-15$ being coated with a $5 \mathrm{~mm}$ length of silica. RF magnetron sputtering at $200 \mathrm{~W}$ from a $200 \mathrm{~mm}$ diameter pure silica target was employed, with the substrate at ambient temperature in a $10 \%$ oxygen atmosphere at a total pressure of $11 \mathrm{~m}$ Torr for 12 hours. It is clear that annealing for 1 hour at $250^{\circ} \mathrm{C}$ substantially improves the transmission of the coated waveguides and that no further measurable improvement is obtained after 2 hours annealing. Following these preliminary measurements, a silica isolation layer of approximate thickness $1 \mu \mathrm{m}$ was sputtered onto the sensor chips in a two-stage process, with the windows of $8 \mathrm{~mm}$ and $0.8 \mathrm{~mm}$ length being defined photolithographically by positive lift off. After definition of the photoresist layer, a high-transparency film approximately $500 \mathrm{~nm}$ thick was deposited using the conditions above. To render the isolation layer less porous, a further $500 \mathrm{~nm}$ silica was then deposited, without removing the sample from the chamber, by sputtering in pure argon for 4 hours. The devices were then annealed in air at $250^{\circ} \mathrm{C}$ for 1 hour. The first layer is sufficiently thick to 
optically isolate the waveguides from the more highly absorbing second layer, which exhibits reduced porosity and prevents penetration of liquids towards the waveguide surface.

3.3 Tantalum pentoxide film. After initial optical measurements, tantalum pentoxide films were deposited over the entire surfaces of the chips by reactive RF sputtering from a tantalum target, in an atmosphere containing $10 \%$ oxygen at a total pressure of $11 \mathrm{mTorr}$. Sensor chip 1, to be used to demonstrate the operating principles, was coated with a $\mathrm{Ta}_{2} \mathrm{O}_{5}$ film of thickness $25 \pm 1 \mathrm{~nm}$. Sensor chip 2, to be used to study the effect of $\mathrm{Ta}_{2} \mathrm{O}_{5}$ film thickness variation, was coated with a film deposited in three stages (each of thickness $15 \pm(\mathrm{nm})$. For this chip, sensing measurements were conducted before and after each stage, so that results were obtained for the same MZIs with a $\mathrm{Ta}_{2} \mathrm{O}_{5}$ overlayer of nominal cumulative thickness $0,15,30 \& 45 \mathrm{~nm}$. The final device, with the $45 \mathrm{~nm} \mathrm{Ta}_{2} \mathrm{O}_{5}$ overlayer, was then annealed in air at $250^{\circ} \mathrm{C}$ for 1 hour, and the measurements repeated.

\section{Measurement procedures}

Characterisation of device performance as a refractometer was carried out with liquids of known refractive index, using the configuration shown in Figure 1. Light from a $10 \mathrm{~mW}$ $633 \mathrm{~nm}$ He-Ne laser was coupled into a single-mode polarisation-maintaining fibre (Fibercore), which was butt-coupled to the sensor chip. A preliminary inspection confirmed that the waveguides were monomode at this wavelength. $\mathrm{A} \times 10$ microscope objective lens was used to focus the output signals on a 1D CCD array (Toshiba), which comprised 3648 pixels of width $8 \mu \mathrm{m}$ and height $200 \mu \mathrm{m}$. A $40 \mathrm{~dB}$ neutral density filter was placed between the lens and the detector to reduce the background radiation incident on the CCD array while attenuating the signal to avoid saturating the CCD; a sheet polariser was used to select the TE polarisation. A flow-cell was clamped to the sensor surface and test solutions of sucrose in water were applied using a flow-injection system. For sensor chip 1, a sucrose solution of 
index varying gradually from $n=1.3330$ to $n=1.3832$ over 30 minutes was passed over the sensor surface, to illustrate the operation of the device. Subsequently, measurements were made on sensor chip 2 by injecting solutions of known index into the background stream of water, to calibrate the device. No attempt was made to control the temperature at the sensor surface. The refractive indices of the sucrose solutions were determined using a commercial refractometer (Kyoto Electronics RA-510) at a wavelength of $589 \mathrm{~nm}$ at $20^{\circ} \mathrm{C}$.

A typical 1D spatial distribution of the optical intensity falling on the CCD array is shown in Figure 3 for the device in air. The eight outputs of the device are clearly visible and well separated, and are labelled according to the device layout in Figure 1. The subscripts $\mathrm{L}$ and $\mathrm{H}$ indicate the low- and high-sensitivity MZI respectively while Ref $_{1}$ refers to the output of the reference waveguide exposed to the analyte for $8 \mathrm{~mm}$, and $\operatorname{Ref}_{2}$ refers to the output isolated from the analyte for its entire length. The inset shows an expanded view of one output distribution. The intensity of $\operatorname{Ref}_{1}$ is higher than that of $\operatorname{Ref}_{2}$ either because of the attenuation due the additional $8 \mathrm{~mm}$ of silica overlaying it or because of imperfections in the waveguides. The power emerging from each output was determined as the sum of the signals from each pixel within a 400 micron region surrounding each feature, after subtraction of a background signal obtained in a similar way from a 400 micron region close to each feature. The integration time for each measurement was $20 \mathrm{~ms}$; for each data point within a test cycle ten such integrated signals were collected and averaged.

\section{Experimental results}

5.1 Temporal response to slowly varying index. Figure 4 shows the temporal response of all the waveguide outputs for sensor chip 1 , coated with $25 \mathrm{~nm} \mathrm{Ta}_{2} \mathrm{O}_{5}$, when an aqueous sucrose solution of gradually varying index was passed through the flow-cell at a constant flow rate. The index varied from $n=1.3330$ to $n=1.3832$ over 30 minutes. Figure 4a shows 
the results for the three outputs of the low-sensitivity MZI, Figure $4 \mathrm{~b}$ shows the simultaneous results for the high-sensitivity MZI, and Figure $4 \mathrm{c}$ shows the behaviour of the reference outputs. Each output is normalised to the isolated reference output, $\operatorname{Ref}_{2}$, to remove the effects of input power fluctuations, so that the output plotted for $\operatorname{Ref}_{2}$ is always unity. The low-sensitivity MZI shows that for an index change, $\Delta \mathrm{n}$, of $5 \times \mathrm{D}^{-2}$ a phase change greater than $2 \pi$ radians is induced in the $0.8 \mathrm{~mm}$ window. Outputs $1 \mathrm{~L}$ and $3 \mathrm{~L}$ carry significant power and change significantly while output $2 \mathrm{~L}$ carries little power. As described in Reference 9 this is because the $3 \times 3$ coupler is not strongly coupled, so that for these waveguide fabrication conditions, the inter-waveguide spacing in the coupler should be reduced for improved performance. The high-sensitivity MZI exhibits similar behaviour except that the output passes through nearly 14 interference cycles; as expected, the phase change is ten times greater than that of the low-sensitivity MZI. The data from the lowsensitivity MZI removes the need to count the number of cycles experienced by the highsensitivity MZI, which yields the precise index data. The output signals reach equilibrium approximately 1500 s after pulse injection when full mixing in the flow-cell has been obtained. It is clear from Figures $4 \mathrm{a}$ and $4 \mathrm{~b}$ that the interferometer contrast is reduced as the analyte refractive index increases. This is due to the additional loss induced in the measurement windows of the MZIs at higher analyte indices due to enhancement of the light intensity in the lossy tantalum pentoxide film. This is confirmed by the $\operatorname{Ref}_{1}$ output in Figure $4 \mathrm{c}$ where the transmission of this waveguide, which is coated with $8 \mathrm{~mm} \mathrm{Ta}_{2} \mathrm{O}_{5}$ in common with the sensing region of the high-sensitivity MZI, is seen to fall as the index increases.

5.2 Device sensitivity. Figure 5 shows the phase change introduced, for sensor chip 2, in the high-sensitivity MZI window against refractive index for the range of sucrose solutions tested, for $\mathrm{Ta}_{2} \mathrm{O}_{5}$ thicknesses of $0,15 \& 30 \mathrm{~nm}$, with the phase set at zero for water. The losses induced in the high-sensitivity $\mathrm{MZI}$ by the $45 \mathrm{~nm}$ thick $\mathrm{Ta}_{2} \mathrm{O}_{5}$ film were too high to 
allow measurements to be made, so that the results for the low-sensitivity MZI are shown for this film thickness. These data were acquired by injecting "pulses" of aqueous sucrose solution of known refractive index into a flowing stream of water of index 1.3330. The phase change was determined using a multiple curve-fitting algorithm from traces similar to, but more rapid than, those shown in Figure 4. It can be seen that the addition of the $\mathrm{Ta}_{2} \mathrm{O}_{5}$ film to the high-sensitivity MZI has significantly increased the sensitivity of the device in an aqueous medium. The sensitivity is also seen to increase with increasing analyte index, as a greater proportion of modal power is carried in the analyte for higher indices. The sensitivities, deduced from the average slopes of the fitted curves, range from 35 radians per unit refractive index (RIU) for the uncoated device to 95 radians/RIU and $210 \mathrm{radians} / \mathrm{RIU}$ for the $15 \mathrm{~nm}$ and $30 \mathrm{~nm}$ coated devices respectively, representing enhancement by a factor of 2.75 and 6, respectively. The low-sensitivity MZI with the $45 \mathrm{~nm} \mathrm{Ta}_{2} \mathrm{O}_{5}$ film showed a phase sensitivity of 305 radians/RIU, corresponding to an enhancement by a factor of 8.75 compared with the uncoated high-sensitivity MZI. In terms of enhancement in sensitivity per unit index per unit window length, this corresponds to an enhancement by a factor of 87.5 compared with an uncoated MZI. Table 1 gives the complete set of sensitivities and sensitivity enhancement factors of the four $\mathrm{Ta}_{2} \mathrm{O}_{5}$ thicknesses. In the case of the $45 \mathrm{~nm}$ film " $\times 10 "$ denotes the projected sensitivity and enhancement for the high-sensitivity MZI had it been measureable. The detection limit, estimated by comparison of the sensitivity with the phase resolution of the measurement system with water flowing over the sensor, is also given. For example, a phase resolution of $5 \times 10^{-3}$ radians $\left(0.3^{\circ}\right)$ was deduced from the system noise for devices with $30 \mathrm{~nm}$ or less $\mathrm{Ta}_{2} \mathrm{O}_{5}$, resulting in a minimum detectable refractive index change of $2.6 \times 10^{-5}$ for $30 \mathrm{~nm} \mathrm{Ta}_{2} \mathrm{O}_{5}$. The signal to noise ratio was found to have degraded strongly for the device with $\mathrm{Ta}_{2} \mathrm{O}_{5}$ thickness of $45 \mathrm{~nm}$, resulting in a poorer detection limit. 
5.3 Attenuation in sensing window. In Figure $4 \mathrm{c}$ the output of waveguide $\operatorname{Ref}_{1}$ on sensor chip 1 shows that the attenuation due to the $8 \mathrm{~mm}$ window, including the transitions from the waveguide overlaid with silica to that overlaid with $25 \mathrm{~nm} \mathrm{Ta}_{2} \mathrm{O}_{5}$ and the propagation losses due to roughness and absorption in the $\mathrm{Ta}_{2} \mathrm{O}_{5}$, is significant in water and increases with increasing analyte index. As analyte index increases, the modal fields in the $\mathrm{Ta}_{2} \mathrm{O}_{5}$-coated region are drawn towards the waveguide surface, increasing the modal mismatch between the coated and uncoated regions and causing more power to propagate in the relatively lossy $\mathrm{Ta}_{2} \mathrm{O}_{5}$ film; both these effects serve to increase attenuation. Such attenuation will also cause reduced interference contrast in the MZIs, acting against the increased phase sensitivity obtained with thicker $\mathrm{Ta}_{2} \mathrm{O}_{5}$ films to reduce the minimum detectable index change. This additional attenuation in the reference arm for sensor chip 2 is plotted against analyte index in Figure 6, for all the $\mathrm{Ta}_{2} \mathrm{O}_{5}$ thicknesses used and, in addition, for the $45 \mathrm{~nm}$ thick $\mathrm{Ta}_{2} \mathrm{O}_{5}$ film after annealing in air at $250^{\circ} \mathrm{C}$ for 1 hour. The results show that over the index range of interest the attenuation is insignificant for the device without $\mathrm{Ta}_{2} \mathrm{O}_{5}$ coating and for that with a $15 \mathrm{~nm}$ film. The window attenuation becomes significant with $30 \mathrm{~nm} \mathrm{Ta}_{2} \mathrm{O}_{5}$, and becomes catastrophic for $45 \mathrm{~nm} \mathrm{Ta}_{2} \mathrm{O}_{5}$, with attenuation of over $30 \mathrm{~dB}$ for all analyte indices above that of water. Annealing the device reduces the attenuation significantly, probably by reducing the optical absorption of the $\mathrm{Ta}_{2} \mathrm{O}_{5}$, but it is still excessive for successful operation of the high-sensitivity MZI.

\section{Discussion}

The modal intensity distributions in Figure 3 show that multiple interferometers and reference channels can readily be integrated on a single chip and conveniently and flexibly interrogated using input fibre coupling and a linear CCD array. It is clear from Figure 4, however, that while the two MZIs show a factor of 10 difference in sensitivity to index 
changes, as expected, there are significant non-idealities in their responses. The first is that the attenuation in the sensing window increases with analyte index, resulting in reduced contrast in the interference functions, and in the waveguide output powers being more complex functions of analyte index than expected. The attenuation is due largely to absorption and roughness in the $\mathrm{Ta}_{2} \mathrm{O}_{5}$ film and may be reduced by annealing, as shown in Figure 6. Further optimisation of the $\mathrm{Ta}_{2} \mathrm{O}_{5}$ sputtering conditions is expected to lead to further reductions in the component of attenuation due to roughness [12]. However, the output powers are a repeatable function of analyte index and, after calibration, a multiple curve-fitting algorithm can readily extract the index from the data. The second non-ideality is that the three interferometer outputs are not shifted by $120^{\circ}$ with respect to each other and the central outputs of the $3 \times 3$ couplers carry little power, due to weak coupling [10]. This will result in there being regions of analyte index where the overall sensitivity to small changes in index is reduced, but slight modification of the waveguide separations in the coupler would resolve this problem.

Figure 5 shows that adding a thin $\mathrm{Ta}_{2} \mathrm{O}_{5}$ film over the window of the MZI substantially increases the sensitivity of the interferometer to changes in analyte index, with the sensitivity enhancement increasing rapidly with $\mathrm{Ta}_{2} \mathrm{O}_{5}$ thickness. It also shows that the sensitivity increases with analyte index in all cases. Both these effects are due to enhancement of the proportion of modal power traveling in the analyte [9]. The sensitivity of the device coated with the $30 \mathrm{~nm}$ film is less than that reported in Reference 9, for the same film thickness, due to a substrate with a lower sodium concentration being used, resulting in lower $\Delta n$ waveguides, and due to the shorter length of the sensing region. However, BK-7 rather than soda-lime glass was chosen for this work due to its ready availability and reproducible characteristics, and $8 \mathrm{~mm}$ sensing windows were chosen to realise a reasonably compact 
device. It is expected that a slightly thicker $\mathrm{Ta}_{2} \mathrm{O}_{5}$ film would compensate for these differences.

Measurements could not be made on the high-sensitivity MZI when coated with $45 \mathrm{~nm}$ $\mathrm{Ta}_{2} \mathrm{O}_{5}$, due to high losses in the sensing window, as confirmed in Figure 6. However, from measurements made on the low-sensitivity MZI when coated with $45 \mathrm{~nm} \mathrm{Ta}_{2} \mathrm{O}_{5}$, we may deduce an enhancement in phase sensitivity due to a $45 \mathrm{~nm} \mathrm{Ta}_{2} \mathrm{O}_{5}$ film of a factor of more than 80 . Unfortunately the attenuation caused by this film does not allow the use of "highsensitivity" MZIs with $8 \mathrm{~mm}$ long sensing windows. This attenuation is due in part to absorption and scattering losses in the $\mathrm{Ta}_{2} \mathrm{O}_{5}$ film, which may be reduced as described above. However, for this thickness of film, the intensity distribution of the mode in the $\mathrm{Ta}_{2} \mathrm{O}_{5}$-coated waveguide is very different from that in the silica-coated waveguide leading up to it, causing a very substantial loss in power both at the transition into the $\mathrm{Ta}_{2} \mathrm{O}_{5}$-coated region and at out of it, due to poor modal overlap. A homogeneous slab waveguide model approximating a similar waveguide system at this wavelength has predicted that the loss for a single transition would be approximately $1.5 \mathrm{~dB}$ for a $30 \mathrm{~nm} \mathrm{Ta}_{2} \mathrm{O}_{5}$ film and $7 \mathrm{~dB}$ for a $45 \mathrm{~nm}$ $\mathrm{Ta}_{2} \mathrm{O}_{5}$ film with a water superstrate [13]. There is therefore a compromise, in finding the thickness which results in the lowest detection limit, between increasing phase sensitivity and increasing attenuation as the $\mathrm{Ta}_{2} \mathrm{O}_{5}$ thickness increases, even if the propagation losses can be reduced.

The noise on the normalised output signals has been analysed and it is estimated that, for the integration times and averaging procedures used here, the phase resolution is $0.3^{\circ}$ for the device coated with a $\mathrm{Ta}_{2} \mathrm{O}_{5}$ film of thickness of $30 \mathrm{~nm}$ or less. This corresponds to a minimum detectable analyte index change of $2.6 \times 10^{-5}$ for a device operating in a sensitive region of its characteristic (or with an optimised $3 \times 3$ coupler) or a change in the modal 
effective index of approximately $6 \times 10^{-8}$. The minimum resolvable phase change for the lowsensitivity device coated with $45 \mathrm{~nm} \mathrm{Ta}_{2} \mathrm{O}_{5}$ increased to $1.7^{\circ}$, due to the attenuation and reduced contrast. Therefore, although this showed the greatest measured sensitivity in terms of phase, it did not show the lowest detection limit. It is expected that the optimum $\mathrm{Ta}_{2} \mathrm{O}_{5}$ film thickness for this waveguide system and wavelength falls between 30 and $45 \mathrm{~nm}$. Further improvements in detection limit could be achieved by decreasing the bandwidth of the detection system, and by permanently pig-tailing the input fibre to the sensor chip, to reduce mechanically induced fluctuations.

\section{Conclusions}

The fabrication and operation of an optical fibre coupled dual-sensitivity integrated optical Mach-Zehnder Interferometer sensor chip incorporating 3x3 directional coupler combiners and internal referencing is described. Chips coated with five different thicknesses of highindex $\mathrm{Ta}_{2} \mathrm{O}_{5}$ film, in order to enhance sensitivity, were fabricated and measurements of their response to liquid analyte index were carried out by passing aqueous sucrose solutions over the sensor surface using a flow injection analyser. The use of these films is shown to increase sensitivity by a factor of six with a film thickness of $30 \mathrm{~nm}$. The chip design allows precise measurements of small index changes without ambiguity over a wide range of indices, and fine-tuning of the design, fabrication and instrumentation is expected to yield further improvements in detection limit. These devices are expected to find application in multi-purpose instrumentation for chemical sensing and biosensing due to their robustness, high sensitivity, and tolerance to the optical properties of surface layers used to add specificity.

Acknowledgments The authors gratefully acknowledge David Sager for assistance with fabrication. 


\section{References}

[1] P.V. Lambeck, Integrated optical sensors for the chemical domain, Proc. $10^{\text {th }}$ European Conference on Integrated Optics, Paderbom, Germany, Apr. 4-6 (2001) pp. 153-158.

[2] K. Itoh \& M. Madou, Optical waveguides for surface spectroscopy $-\mathrm{FePO}_{4}$ thin-film $\mathrm{K}^{+}$doped glass composite optical waveguide systems having tapered velocity couplers, J. Appl. Phys. 69 (1991) pp. 7425-7429.

[3] C. Piraud, E.K. Mwarania, G. Wylangowski, K. O’Dwyer, J. Wilkinson \& D.J.Schiffrin, Optoelectrochemical thin-film chlorine sensor employing evanescent fields on planar optical waveguides, Anal. Chem. 64 (1992) pp. 651-655.

[4] G. Gauglitz \& J. Ingenhoff, Design of new integrated optical substrates for immunoanalytical applications, Fresen. J. Anal. Chem. 349 (1994) pp. 355-359.

[5] R. Kherrat, N. JaffrezicRenault, P. Greco, H. Helmers, P. Benech, R. Rimet, Sens. Actuat. B-Chem 37 (1996) pp. 7-11.

[6] W. Lukosz, Principles and sensitivities of integrated optical and surface plasmon sensors for direct affinity sensing and immunosensing, Biosens. Bioelectron. 6 (1991) pp. 215-225.

[7] R.G.Heideman \& P.V. Lambeck, Remote opto-chemical sensing with extreme sensitivity: design, fabrication and performance of a pigtailed integrated optical phasemodulated Mach Zehnder interferometer system, Sens. Actuat. B-Chem 61 (1999) pp. 100127. 
[8] J.C.Abanulo, R.D.Harris, P.N.Bartlett \& J.S.Wilkinson, Waveguide surface plasmon resonance study of electrochemically generated oxide layer on gold, Appl. Opt. 40 (2001) pp. 6242-6245.

[9] G.R.Quigley, R.D.Harris \& J.S.Wilkinson, Sensitivity enhancement of integrated optical sensors by thin high index films, Appl. Opt. 38 (1999) pp. 6036-39.

[10] B.J.Luff, J.S.Wilkinson, J.Piehler, U.Hollenbach, J.Ingenhoff \& N.Fabricius, Integrated optical Mach-Zehnder biosensor", J. Lightwave Technol. 16 (1998) pp. 583-592.

[11] A. Miliou, H. Zhenguang, H.C. Cheng, R. Srivastava \& R.V. Ramaswamy, Fibercompatible $\mathrm{K}^{+}-\mathrm{Na}^{+}$ion-exchanged channel wave-guides - fabrication and characterization, IEEE J. Quantum Elect. 25 (1989) pp. 1889-1897.

[12] J.M. Bennett, M.M. Tehrani, J. Jahanmir, J.C.Podlesny \& T.L Balter, Topographic measurements of supersmooth dielectric films made with a mechanical profiler and scanning force microscope, Appl. Opt. 34 (1995) pp.209-212.

[13] G.R. Quigley, Integrated optical multisensors for water quality, $\mathrm{PhD}$ thesis, Southampton University, UK, (2000). 
Ping Hua received the BSc in physics in 1982 from Suzhou University, Suzhou, China. From 1982 to 1989 she was a physics teacher at the Technical College, Wuxi, China. Since 1990 she has been with the Optoelectronics Research Centre (ORC) at Southampton University. Her research interests include integrated optical waveguide-based sensors applied to biology, chemistry and environmental monitoring systems. She is currently working on the European Union Automated Water Analyser Computer Supported System (AWACSS) project.

B. Jonathan Luff received the $\mathrm{PhD}$ in the field of optoelectronics in 1989 from the University of Sussex. After postdoctoral work at Sussex University he joined the Optoelectronics Research Centre at Southampton University as a Research Fellow in 1993, working on integrated optical devices for sensing applications. He is now with Bookham Technology plc.

Geoffrey R Quigley received a BSc in Physics from Imperial College in 1995 and a PhD in the field of integrated optics in 2001 from the Optoelectronics Research Centre, University of Southampton. The main thrust of his $\mathrm{PhD}$ research was investigating the use of thin high index films to increase the sensitivity of evanescent wave sensors. He is now working for Autonomy Systems Ltd in Cambridge as Wireless Product Manager.

Kenji Kawaguchi received the degree of Master of Electronics Engineering in the field of the blood flow measurement for artificial hearts in 1981 from the University of Kyoto Institute of Téchnology. He is now working for Kyoto Electronics Manufacturing Co.,Ltd (KEM) and researching and developing sensors and analytical instruments for chemical and/or physical quantities. He has 15 patents and more than 20 pending patents in this field. He was seconded to the Optoelectronics Research Centre for collaborative research work from 1996 to 1999 . He is now a general manager and a senior researcher of the Kyoto 
laboratory of KEM.

James Wilkinson received the $\mathrm{BSc}(\mathrm{Eng})$ in electronics in 1977 and the $\mathrm{PhD}$ in the field of integrated optics in 1985, both from University College London. From 1977 to 1979 he was with the GEC Hirst Research Centre working on optical fibre telecommunications systems. From 1983 to 1985 he was with the Department of Nephrology of St Bartholomew's Hospital, London, working on sensing and control for haemodialysis procedures. He is now Professor of Optoelectronics in the Department of Electronics \& Computer Science, University of Southampton, UK. He is seconded to the Optoelectronics Research Centre, where his research group investigates integrated optical devices including chemical and biochemical sensors with particular application to water quality monitoring, and integrated lasers and amplifiers. 


\begin{tabular}{|c|c|c|c|}
\hline $\begin{array}{c}\mathrm{Ta}_{2} \mathrm{O}_{5} \\
\text { thickness } \\
(\mathrm{nm})\end{array}$ & $\begin{array}{c}\text { Sensitivity } \\
\text { (Radians/RIU) }\end{array}$ & $\begin{array}{c}\text { Sensitivity } \\
\text { enhancement } \\
\text { factor }\end{array}$ & $\begin{array}{c}\text { Detection } \\
\text { limit } \\
\text { (RIU) }\end{array}$ \\
\hline 0 & 35 & 1 & $1.5 \times 10^{-4}$ \\
\hline 15 & 95 & 2.75 & $5.5 \times 10^{-5}$ \\
\hline 30 & 210 & 6 & $2.6 \times 10^{-5}$ \\
\hline 45 & $305(\times 10)$ & $8.75(\times 10)$ & $9.6 \times 10^{-5}$ \\
\hline
\end{tabular}

Table 1 Measured sensitivity and detection limit for the high sensitivity MZI $(0,15 \&$ $\left.30 \mathrm{nmTa}_{2} \mathrm{O}_{5}\right)$ and the low-sensitivity $\mathrm{MZI}\left(45 \mathrm{~nm} \mathrm{Ta}_{2} \mathrm{O}_{5}\right)$. 


\section{Figure Captions}

Figure 1. Experimental sensor system.

Figure 2. Effect upon waveguide transmission of annealing silica isolation layer.

Figure 3. Waveguide mode intensity output distributions on linear CCD array.

Figure 4. Temporal response of a) low-sensitivity MZI, b) high-sensitivity MZI and c) reference outputs to varying sucrose solution index.

Figure 5. High-sensitivity $\mathrm{MZI}$ phase change against analyte refractive index for $\mathrm{Ta}_{2} \mathrm{O}_{5}$ film thicknesses from 0 to $30 \mathrm{~nm}$; low-sensitivity MZI phase change against analyte refractive index for $\mathrm{Ta}_{2} \mathrm{O}_{5}$ film thickness of $45 \mathrm{~nm}$.

Figure 6. Attenuation in reference window against analyte index for $\mathrm{Ta}_{2} \mathrm{O}_{5}$ film thicknesses from 0 to $45 \mathrm{~nm}$, and for annealed $45 \mathrm{~nm}$ film. 
Figure 1. Experimental sensor system.

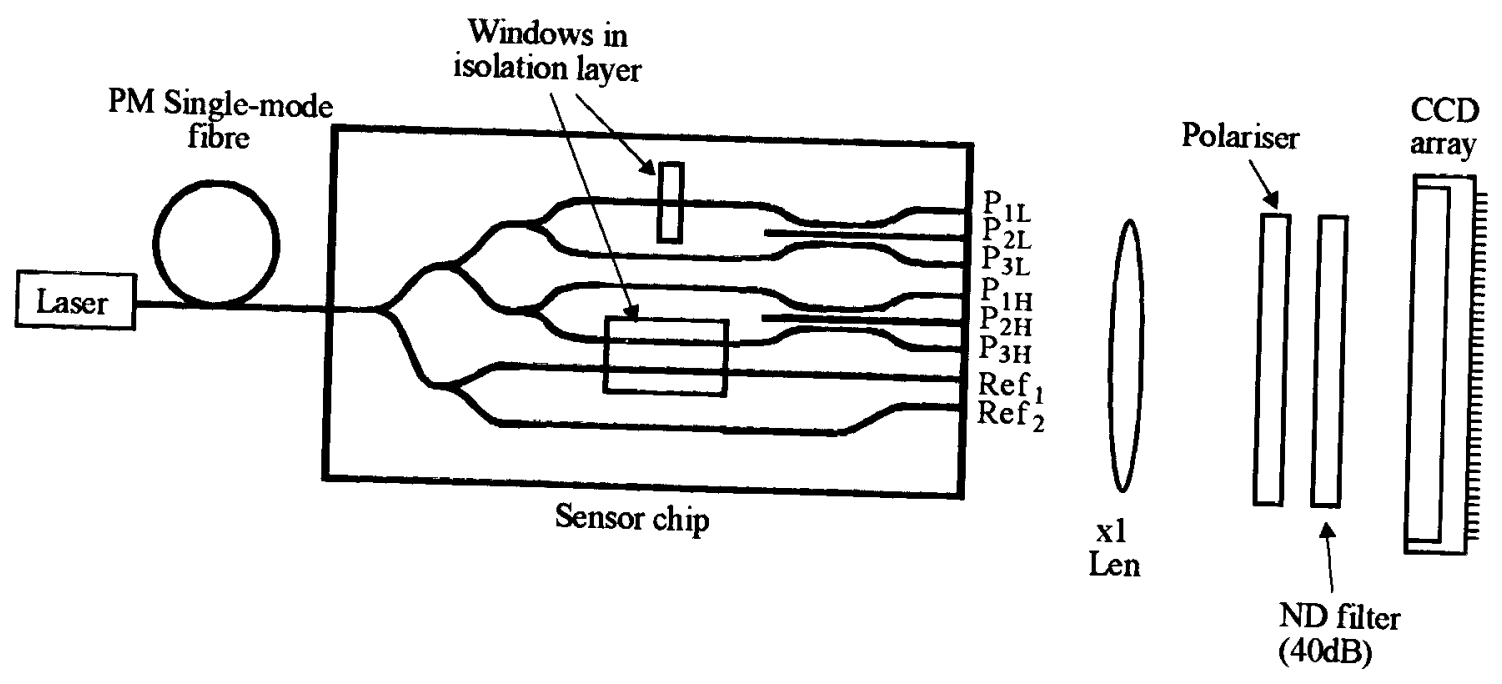


Figure 2. Effect upon waveguide transmission of annealing silica isolation layer.

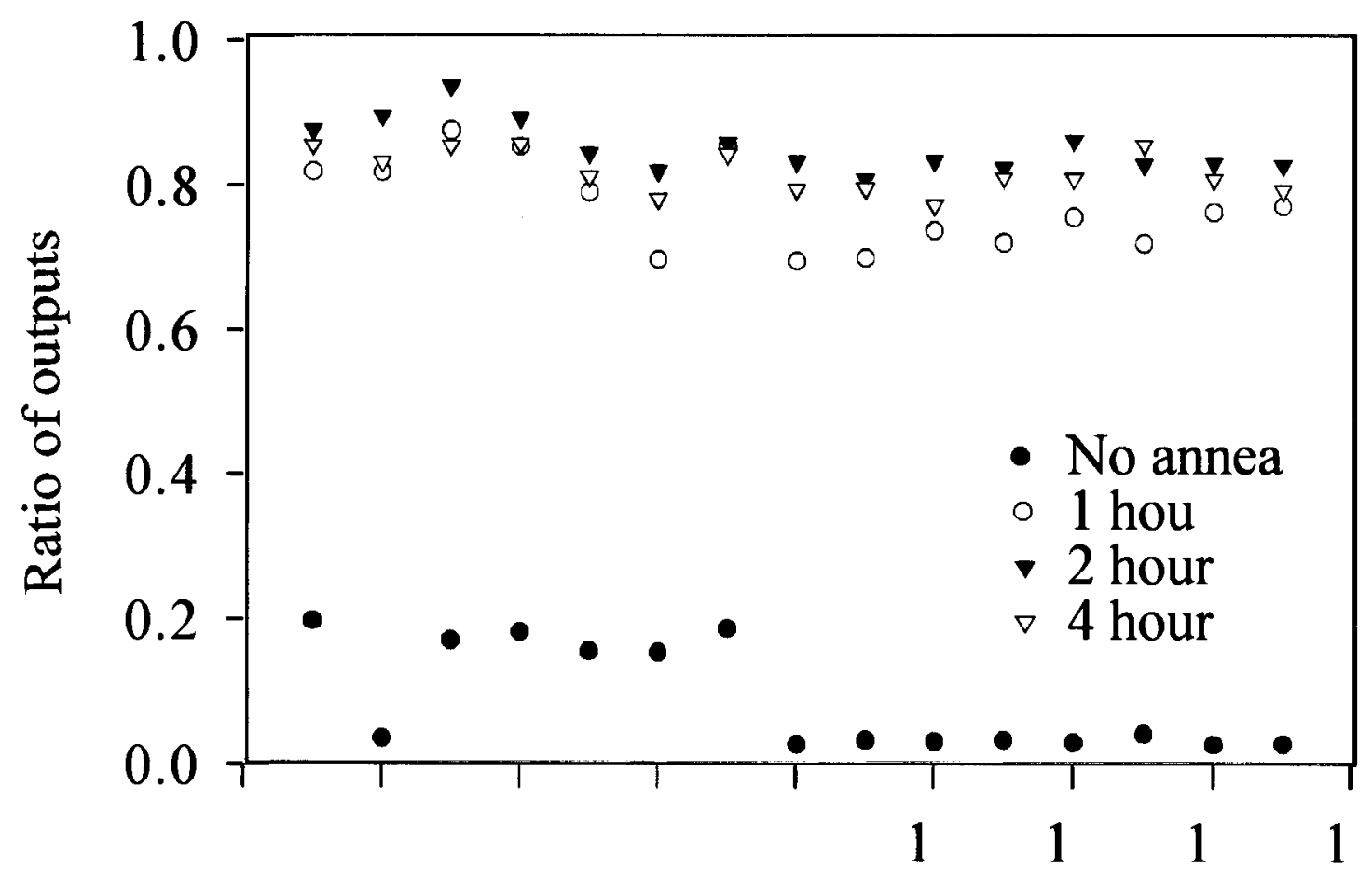

Measurement number 
Figure 3. Waveguide mode intensity output distributions on linear CCD array.

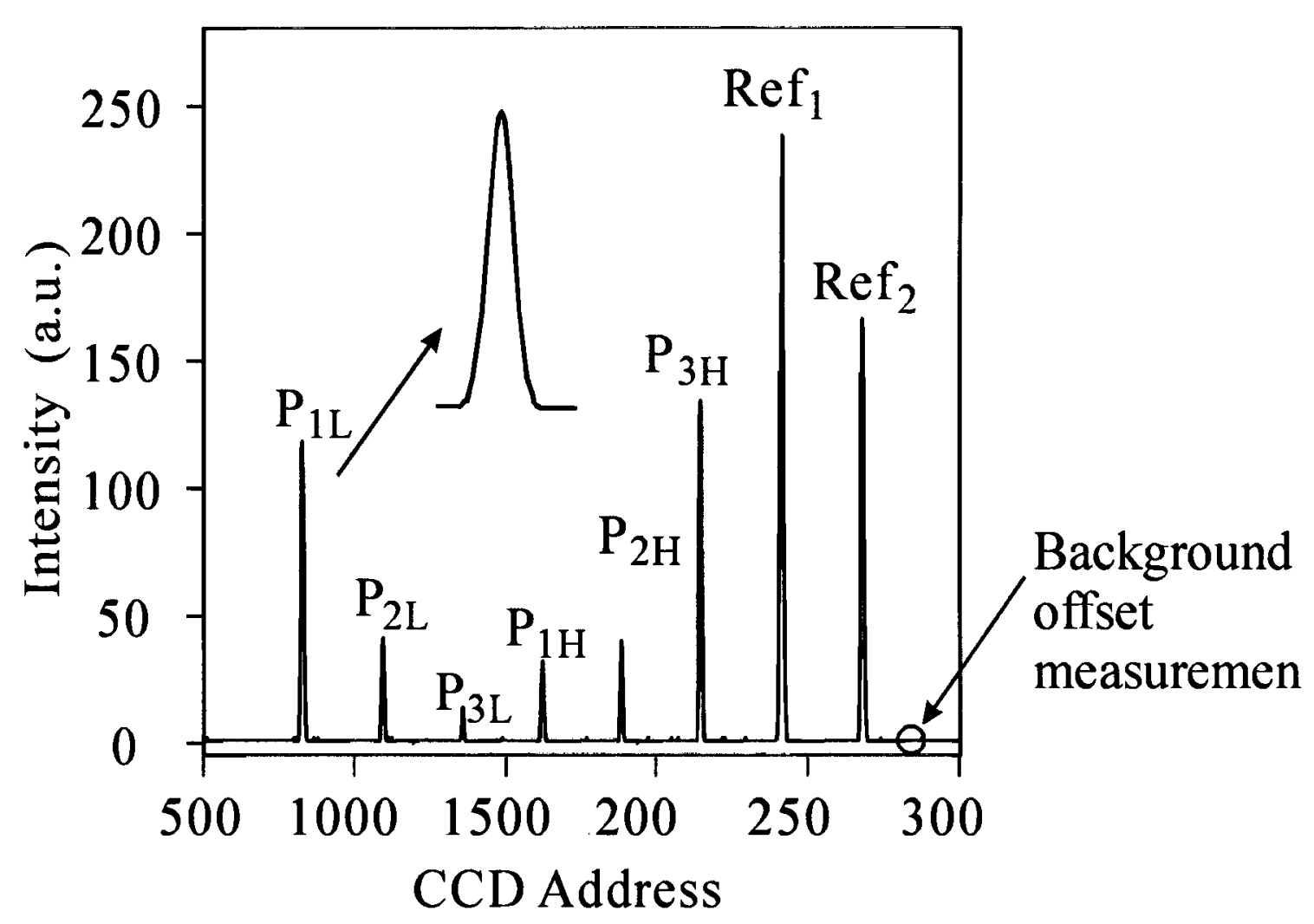


Figure 4. Temporal response of a) low-sensitivity MZI, b) high-sensitivity MZI and c) reference outputs to varying sucrose solution index.
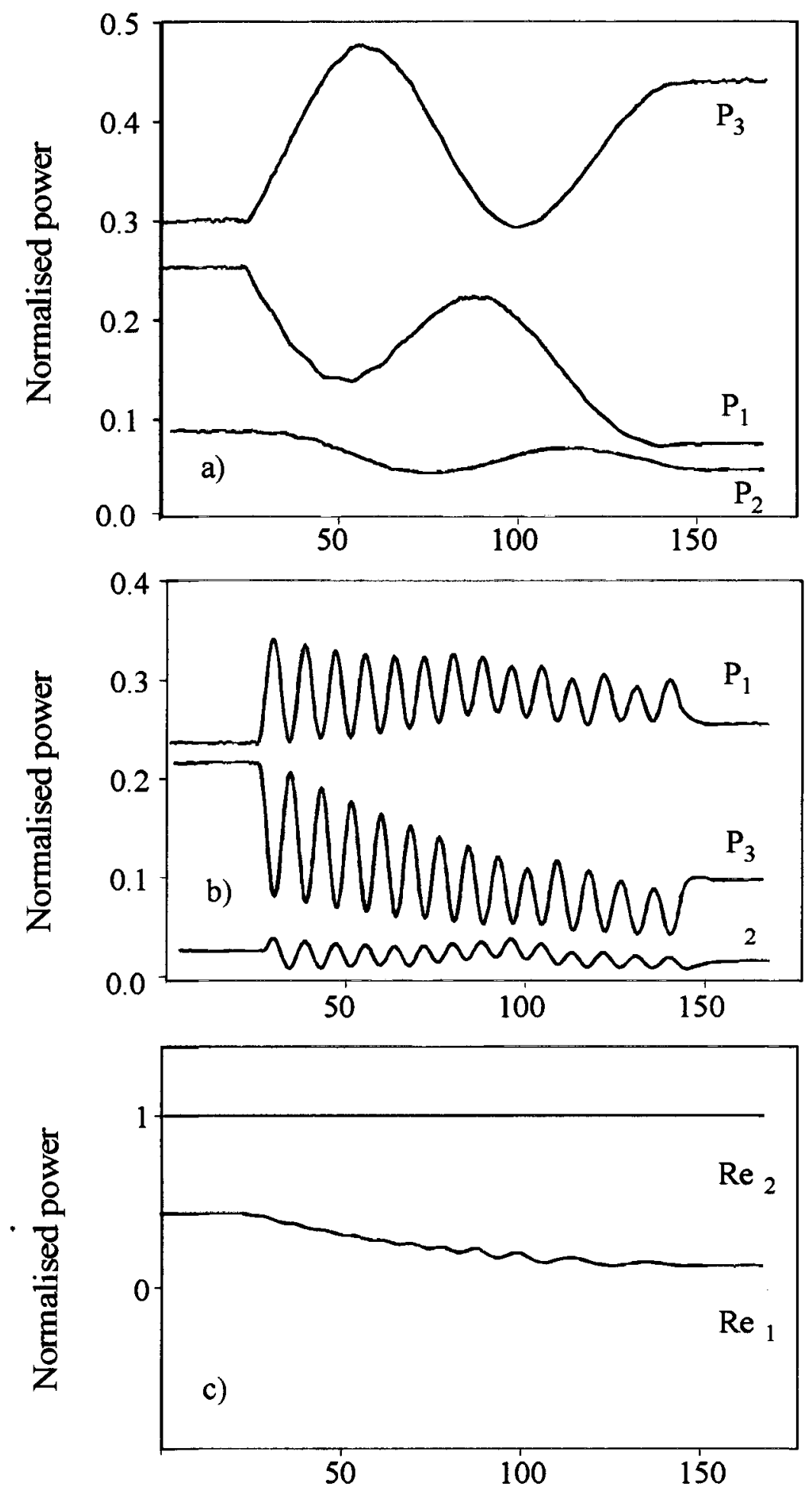

Time $[\mathrm{sec}$ 
Figure 5. High-sensitivity MZI phase change against analyte refractive index for $\mathrm{Ta}_{2} \mathrm{O}_{5}$ film thicknesses from 0 to $30 \mathrm{~nm}$; low-sensitivity MZI phase change against analyte refractive index for $\mathrm{Ta}_{2} \mathrm{O}_{5}$ film thickness of $45 \mathrm{~nm}$.

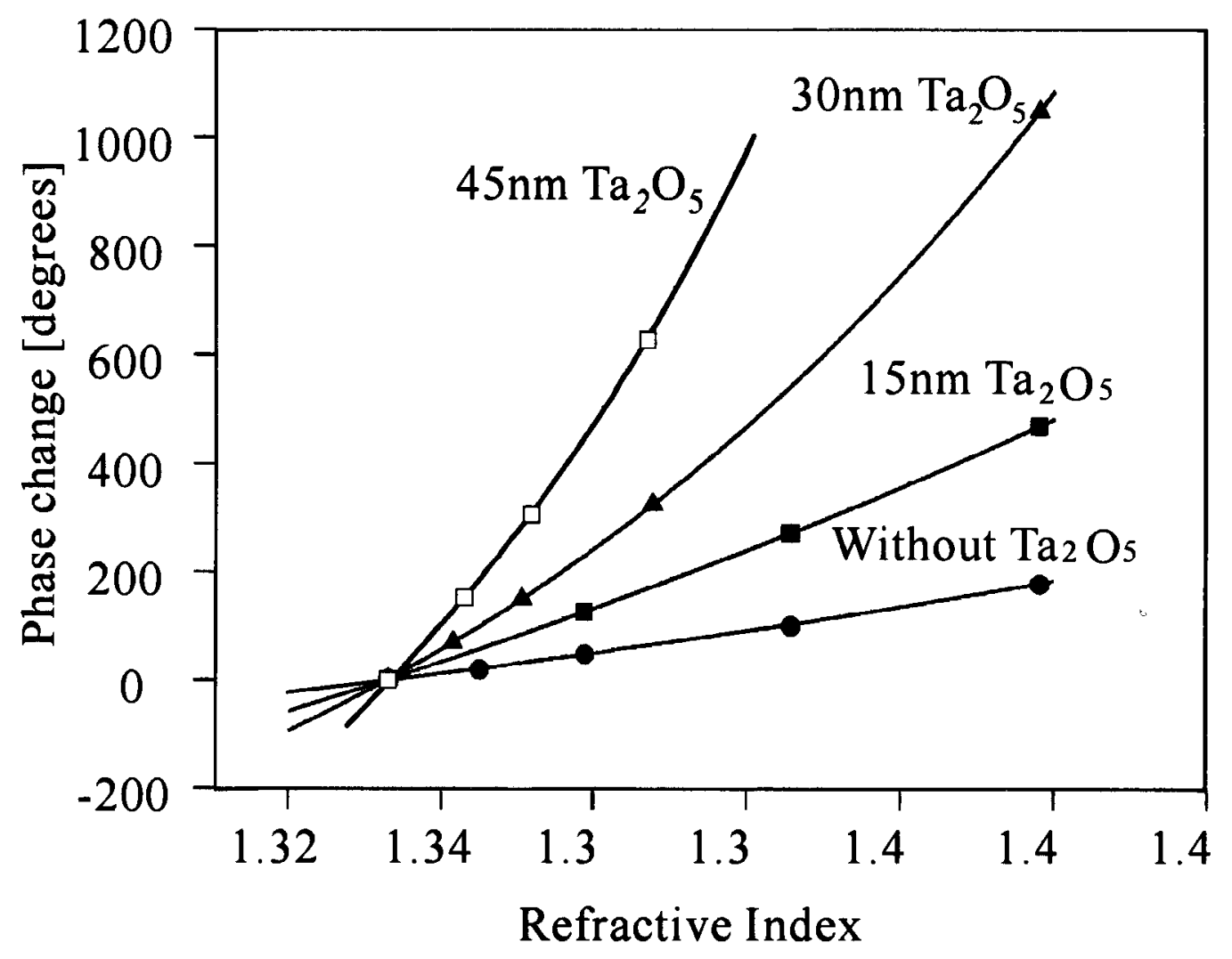


Figure 6. Attenuation in reference window against analyte index for $\mathrm{Ta}_{2} \mathrm{O}_{5}$ film thicknesses from 0 to $45 \mathrm{~nm}$, and for annealed $45 \mathrm{~nm}$ film.

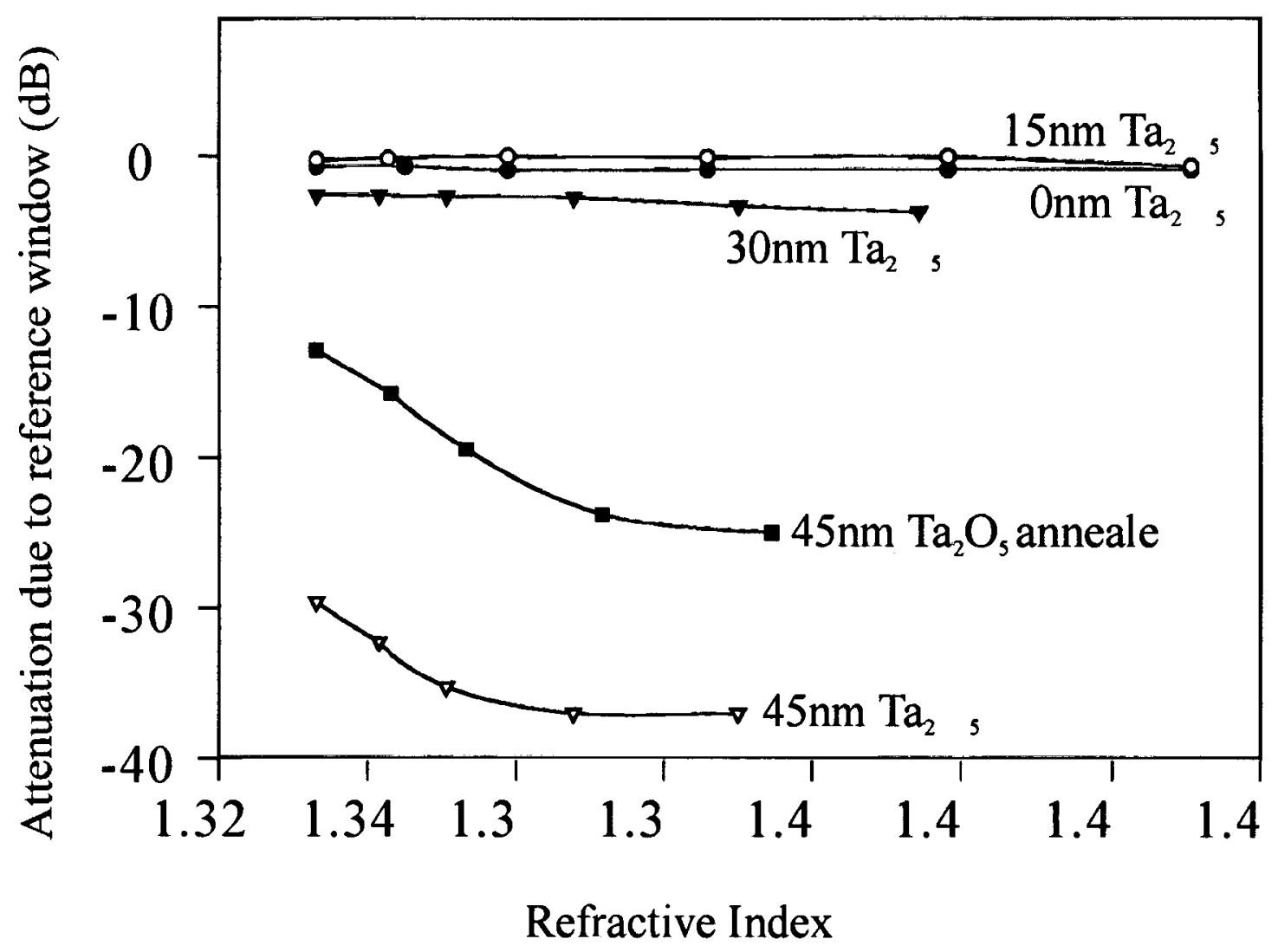

